\title{
Expression of Vimentin in hair follicle growth cycle of inner Mongolian Cashmere goats
}

\author{
Nai Rile ${ }^{1,2,3,4+}$, Zhihong Liu ${ }^{1,2^{*}+}$, Lixia Gao ${ }^{7 \dagger}$, Jingkai Qi ${ }^{\dagger \dagger}$, Meng Zhao ${ }^{6}$, Yuchun Xie ${ }^{1,2}$, Rui Su' ${ }^{1}$ Yanjun Zhang ${ }^{1}$, \\ Ruijun Wang ${ }^{1}$, Jie Li ${ }^{1}$, Hongmei Xiao ${ }^{1}$ and Jinquan $\mathrm{Li}^{1{ }^{*}}$
}

\begin{abstract}
Background: The growth of Inner Mongolian Cashmere goat skin hair follicle exhibits a periodic growth pattern. The hair growth cycle is distinguished as telogen, anagen, and catagen stages. The role of vimentin in the growth process of hair follicles is evident. To elucidate the mechanism underlying the vimentin activity in the growth cycle of hair follicles, transcriptome sequencing and liquid chromatography-tandem mass spectrometry were used to obtain the nucleic acid and amino acid sequences of VIIM gene and vimentin. The amino acid and nucleic acid sequences were analyzed by comparison. Real-time quantitative PCR, Western blot, and immunohistochemistry analyzed the expression level and sites of vimentin in the three growth stages of the Inner Mongolia Cashmere goat skin samples.
\end{abstract}

Results: VIM gene CDNA, obtained by transcriptome sequencing, was aligned against that of the Capra hircus VIM gene. The amino acid sequence of vimentin revealed a high similarity rate across other species. The expressions of both VIM gene and vimentin were highest during the growth period and lowest in the rest period. Furthermore, vimentin was primarily expressed in the outer root sheath of the hair follicle as assessed by staining.

Conclusions: The sequences of the gene and protein are similar to that of other species and identical to Capra hircus. However, the expression of VIM and vimentin was proportional to that of the growth of hair follicles. And vimentin expressed only in the outer root sheath of hair follicles. Thus, vimentin was speculated to participate in the regulation of the hair follicle growth cycle by affecting the outer root sheath.

Keywords: Cashmere goat, Skin, Hair follicle, Growth cycle, Vimentin

\section{Background}

The fleece of Inner Mongolia Cashmere goat can be classified as heterogeneous. Cashmere is a product of the secondary hair follicle and wool is a product of the primary hair follicle. And they undergoes a periodicity comprising of three stages anagen, catagen, and telogen [1]. The periodic changes in the hair follicle are attributed to the complex and orderly biochemical processes including the interaction and inhibition of multimolecule particles in the skin. Thus, the expression level

\footnotetext{
*Correspondence: liuzh7799@163.com; lijinquan_nd@126.com

${ }^{\dagger}$ Equal contributors

${ }^{1}$ College of Animal Science, Inner Mongolia Agricultural University, Hohhot 010018, China

Full list of author information is available at the end of the article
}

of proteins involved in these processes varies correspondingly.

Vimentin is a type III intermediate filament (IF) protein, which is expressed in the whole cytoplasm, except the organelles and nucleus [2, 3]. It is encoded by the VIM gene located on chromosome 13 [4]. The length of the DNA sequence of the VIM gene is approximately $10 \mathrm{~kb}$. The full length of the cDNA is about $1848 \mathrm{bp}$, and the open reading frame is about $1401 \mathrm{bp}$ [5], containing 9 exons that encode 466 amino acids. The protein sequence of vimentin is highly conserved across evolution [5]. The main function of this protein is the maintenance of cell morphology [6-8], signal transmission between cells [9-11], regulation of cell differentiation and proliferation [12,13], and participation in cell 
stress response [14]. Vimentin also plays a major role in the development of tumors $[15,16]$ and viral infections caused by Herpes virus [17], immunodeficiency virus [18], and other viruses. In addition, as an IF protein, vimentin might also be related to the transport of mRNA [19]. A previous study showed that the expression level of vimentin decreased gradually with the growth of a fetal rat skin [20]. Another study proposed that the highest expression of vimentin was observed in the anagen stage of the growth cycle of the mouse skin follicle [21]. Furthermore, a significant difference in vimentin expression levels was observed during the stages of the development cycle of the skin follicle in Inner Mongolia Cashmere goat; the expression was high during the growth period [22]. In summary, vimentin plays a vital role in the growth process of the development cycle of the skin follicle.

\section{Methods}

\section{Animals}

The Experimental Cashmere goats were obtained from Aerbasi White Cashmere Goat Breeding Farm in Inner Mongolia, China. These goats belong to a breed of inner Mongolian Cashmere goats [23]. Three adult individuals (females, 2-year-old) were randomly selected for biological replicates. Skin samples were collected from the back of the goat during the hair follicle developmental stages of telogen (March), anagen (July), and catagen (December). The samples were transported to the laboratory in liquid nitrogen and preserved at $-80{ }^{\circ} \mathrm{C}$.

\section{Determination of mRNA sequence of VIM gene}

The total RNA from the skin tissue was extracted using TRIzol (Takara, Dalian, China). Then, the samples were ground in liquid nitrogen, according to the manufacturer's instructions. The concentration and purity of RNA were detected using a Beckman DU 800 Nucleic Acid-Protein Analyzer (Beckman-Coulter, Fullerton, CA, USA). The integrity of the total RNA was analyzed by $1 \%$ agarose gel electrophoresis. Furthermore, the extracted RNA was synthesized into cDNA (Illumina TruSeq $^{\text {tw }}$ RNA sample preparation kit, USA) for transcriptome sequencing. The next-generation sequencing of the cDNA was performed using an Illumina HiSeq2000 platform; the sequencing length was $2 \times 100 \mathrm{bp}$.

\section{Real-time quantitative PCR}

The cDNA obtained above was used for real-time quantitative PCR (RT-PCR) analysis. Gene-specific primers were designed using the software Primer 3.0 and synthesized by Sangon Biotech Co., Ltd. (Shanghai, China). The primer sequences and fragment size are listed in Table 1. The quantitative reverse transcription RT-PCR was performed using the PrimeScript RT Reagent Kit
Table 1 Primer sequences and fragment size of Cashmere goat VIM gene and $\beta$-actin

\begin{tabular}{llll}
\hline $\begin{array}{l}\text { Gene } \\
\text { Name }\end{array}$ & $\begin{array}{l}\text { GenBank } \\
\text { number }\end{array}$ & Sequence of primer & $\begin{array}{l}\text { Products } \\
\text { size }\end{array}$ \\
\hline VIM & XM_005688054.1 & $\begin{array}{l}\text { F: TTTCTCCACGCCTCCAGTT } \\
\text { R: ATGTCTTCGCCAGGTTGT }\end{array}$ & $296 \mathrm{bp}$ \\
B-actin & NM_001009784.1 & $\begin{array}{l}\text { F: GGCAGGTCATCACCATCGG } \\
\text { R: CGTGTGGCGTAGAGGTCTTT }\end{array}$ & $158 \mathrm{bp}$ \\
& &
\end{tabular}

(TaKaRa) in a $20-\mu \mathrm{L}$ reaction volume with $10 \mu \mathrm{L}$ of $2 \times$ SYBR Premix Ex Taq II (TaKaRa), 0.4 $\mu \mathrm{L}$ ROX, and $0.5 \mu \mathrm{L}$ each primer, and 7.2 $\mu \mathrm{L}$ DNase/RNase-free water. The reaction was assessed on a Bio-Rad IQ5 Multicolor RT-PCR Detection System (Hercules, CA, USA). We selected the $\beta$-actin as the internal contral. The conditions for quantitative RT-PCR are listed in Table 2. The RT-PCR analysis was performed using the $2^{-\Delta \Delta C T}$ method. Statistical analysis was conducted using the GLM method of the SA software (version 9.0; SAS Institute, Cary, NC, USA). The values are represented as the mean \pm standard deviation. A significance level of 0.05 was used.

\section{Acquisition of vimentin sequence and multiple sequence alignment}

An equivalent amount of Inner Mongolia Cashmere goat skin samples were ground in liquid nitrogen, followed by the addition of $1 \%$ sodium dodecyl sulfate(SDS)to the lysate. The mixture was incubated at room temperature for $20 \mathrm{~min}$, followed by ultrasonication for $2 \mathrm{~min}$ and centrifugation at $12,000 \mathrm{rpm}$ for $15 \mathrm{~min}$ at $4{ }^{\circ} \mathrm{C}$. The total protein concentration in the supernatant was determined using the BCA (Bioteke,Bei Jing, China) kit. The protein was hydrolyzed by trypsin, and peptides were analyzed by Triple TOF 5600 (AB Sciex, USA). The protein candidates were searched by software Protein Pilot 4.0. The sequences of vimentin in Capra hircus, Ovis aries, Bos taurus, Homo sapiens, and Mus musculus were obtained from NCBI/Protein and compared to that of NCBI/BLAST. The three-dimensional structure of vimentin was constructed by the comparative protein modeling program SWISS-MODEL.

\section{Western blot}

$0.08 \mathrm{~g}$ of skin samples were homogenized in liquid nitrogen, followed by the addition of cell lysis buffer and

Table 2 Parameters for quantitative RT-PCR

\begin{tabular}{lll}
\hline Temperature & Time & Cycles \\
\hline $94{ }^{\circ} \mathrm{C}$ & $510 \mathrm{~min}$ & \\
$94{ }^{\circ} \mathrm{C}$ & $30 \mathrm{~s}$ & 40 \\
$62^{\circ} \mathrm{C}$ & $30 \mathrm{~s}$ & \\
$72{ }^{\circ} \mathrm{C}$ & $30 \mathrm{~s}$ & \\
\hline
\end{tabular}


PMSF (Beyotime, China) to extract total protein. Subsequently, the tissue samples were placed in an ice bath for ultrasonication, incubated for $30 \mathrm{~min}$, and centrifuged as described above. The total protein was estimated in the resulting supernatant using the BCA kit (Bioteke). An equivalent of $30 \mu \mathrm{g}$ protein was separated by $12 \%$ SDS-polyacrylamide gel electrophoresis (SDS-PAGE), followed by semi-dry transfer to the PVDF membrane (PALL, New York, USA). The PVDF membrane was blocked using $5 \%$ skimmed milk at room temperature for $2 \mathrm{~h}$, followed by probing the membrane with mouse monoclonal anti-vimentin antibody (Abcam, Cambs, UK. 1:500) and rabbit polyclonal anti- $\beta$-actin antibody (Abcam. 1:1,000) overnight at $4{ }^{\circ} \mathrm{C}$. Subsequently, the membrane was washed with phosphatebuffered saline with tween20 (PBST) and incubated with fluorescent-labeled goat anti-mouse secondary antibody and goat anti-rabbit secondary antibody (LI-COR Biosciences Inc., Lincoln, NE, USA. 1: 3,000) at $37^{\circ} \mathrm{C}$ for $1 \mathrm{~h}$. Finally, the membranes were washed, and the immunoreactive bands examined using LI-COR ${ }^{\circ}$ Odyssey nearinfrared imager (LI-COR Biosciences Inc).

\section{Immunohistochemistry}

Fresh skin samples of Inner Mongolia Cashmere goat were obtained during catagen, telogen, and anagen and fixed in $4 \%$ paraformaldehyde for $24 \mathrm{~h}$. Following dehydration in pure alcohol, the sections were placed in benzene to replace ethanol prior to paraffin-embedding. The slices of tissue samples (8- $\mu \mathrm{m}$-thick) were incubated with xylene for dewaxing, subjected to gradient alcohol hydration, and incubated at room temperature in 3\% hydrogen peroxide $\left(\mathrm{H}_{2} \mathrm{O}_{2}\right)$ to inactivate the endogenous catalase. Next, the specimens were flushed with phosphate-buffered saline (PBS), antigen retrieval was carried out in citrate buffer, and blocking with 5\% BSA at room temperature for $1 \mathrm{~h}$. Subsequently, the samples were incubated overnight at $4{ }^{\circ} \mathrm{C}$ with mouse monoclonal anti-vimentin antibody (Abcam. 1:250); 5\% albumin from bovine serum (BSA) was used as a negative control. Then, the samples were washed with PBS and incubated with the HRP-labeled goat anti-mouse secondary antibody ((Beyotime. 1:500) for $1 \mathrm{~h}$ at $37{ }^{\circ} \mathrm{C}$. Finally, the sections were stained with hematoxylin and observed under a microscope.

\section{Results}

\section{Analysis and determination of the mRNA sequence of VIM gene}

The transcriptome sequencing result showed that the length of VIM gene sequence was $1356 \mathrm{bp}$. The results of the sequence analysis using the Genome Browser are illustrated in Fig. 1. The transcript of the VIM gene consists of six exons. The blue color of the figure represents the exons, whereas the pale blue denotes the exon boundary. As shown in Fig. 2, the matching rate between the sequence and the Capra hircus VIM gene from NCBI/GENE was 100\%. The Capra hircus VIM gene begins to coincide with the transcript at the position of $48 \mathrm{bp}$. The squares in Fig. 2 represent the exons, and the lines between them represent the introns. The red color indicates the Capra hircus VIM gene from the NCBI; the blue color represents the sequence position at which the transcriptome sequence is identical to that of the Capra hircus VIM gene.

Differential expression of the VIM gene in the skin follicle growth cycle of inner Mongolian Cashmere goats

In this study, RT-PCR analyzed the differential expression of VIM gene in the skins during the stages of telogen, anagen, and catagen of Inner Mongolia Cashmere goat (Fig. 3). The difference was significant $(P<0.01)$, and the expression level of VIM gene was the highest in the growth period and lowest in the resting period.

\section{Acquisition of vimentin amino acid sequence and multiple sequence alignment}

The length of the amino acid sequence of skin vimentin of Inner Mongolia Cashmere goat was 466 amino acids. Importantly, the length of the vimentin sequence was identical to that with Capra hircus, Ovis aries, Bos taurus, Homo sapiens, and Mus musculus obtained from NCBI/PROTEIN. The sequence alignment using NCBI/ BLAST revealed that the amino acid sequence of vimentin from Inner Mongolia Cashmere goat was 100\% identical to that of the vimentin from Capra hircus, 99\%, 99\%, 98\%, and 97\% identical to that from Bos taurus, Ovis aries, Homo sapiens, and Mus musculus (Fig. 4), respectively. The blue mark in Fig. 4 represents the differentially expressed sequence of vimentin between Inner Mongolia Cashmere goat and Ovis aries. The red mark denotes the site difference in expression from that of Homo sapiens, and the yellow mark represents the site with a difference from the sequence of Mus musculus. The three-dimensional structure of vimentin (144-249 amino acids) was established by the comparative protein modeling program SWISS-MODEL (Fig. 5).

\section{Western blot analysis}

The expression level of vimentin in the skin of Inner Mongolian Cashmere goat was detected by Western blot. The gray-level ratio of the target protein and the internal controls was used as the index for estimating the relative content of the target protein. Fig. $6 \mathrm{a}$ demonstrates the vimentin expression in the skin during all the three periods, and the difference in the expression was extremely significant. Figure $6 \mathrm{~b}$ presents the relative content of vimentin; the expression of vimentin in the 


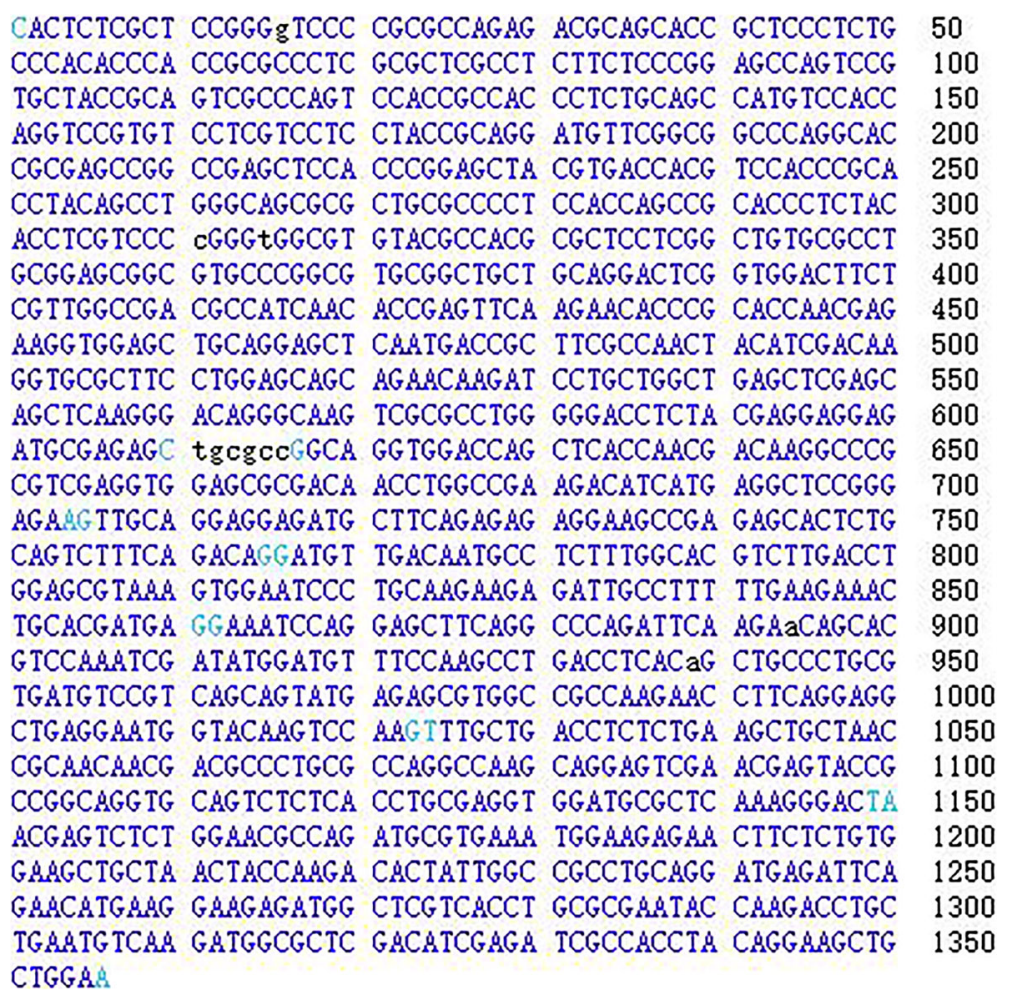

Fig. 1 Transcriptome sequencing result of VIM gene. The transcriptome sequence of VIM gene analysis using Genome Browser shows that the sequence consists of six exons. The pale blue denotes the boundary of each exon

anagen was higher than that in other periods, and the lowest expression was presented during telogen.

\section{Immunohistochemistry}

Immunohistochemistry determined the expression sites of vimentin in the skin of Cashmere goat. These sites were observed under a light microscope, and brown cells were considered to be positive. The immunohistochemistry staining showed that the background staining was colorless or light blue (Fig. 7), and negative results showed no yellow or brown staining. Conversely, the experimental group detected a distinct yellow or brown staining, indicating that the immunohistochemistry method exhibited a specific immune response to vimentin (Fig. 7). The primary expression of vimentin was observed in the outer root sheath (ORS) of the hair follicle. As can be seen from Fig. 7, vimentin was expressed in the ORS in anagen and catagen, and no expression was found in telogen.

\section{Discussion}

The growth cycle of skin follicles in Inner Mongolia cashmere goats

The structure of the hair follicle is composed of a connective tissue sheath, ORS, inner root sheath (IRS), and a hair fiber from the outside to the inside [24]. After the birth of the animal, the skin follicles undergo periodic changes, and the periodic growth of the hair follicle is a complex and highly coordinated system of interaction between epithelial and dermal layers [25]. Previous studies $[26,27]$ showed that in anagen, the epithelial cells of the hair germ grow downwards, as an epidermal finger,

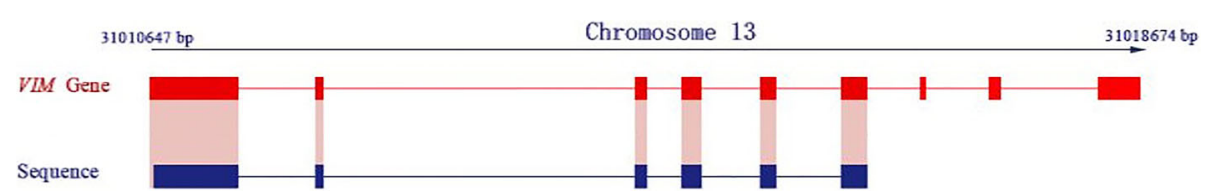

Fig. 2 Comparison between the transcriptome sequencing result of the VIM gene and Capra hircus VIM gene based on the NCBI/GENE. The transcriptome sequence was compared with the Capra hircus VIM gene from the GENE database of NCBI. The boxes in the figure represent the exons, and the lines between them represent the introns. The red color indicates the VIM gene in the NCBI, while the blue color indicates the sequence identical to that of the transcriptome sequence of the VIM gene 


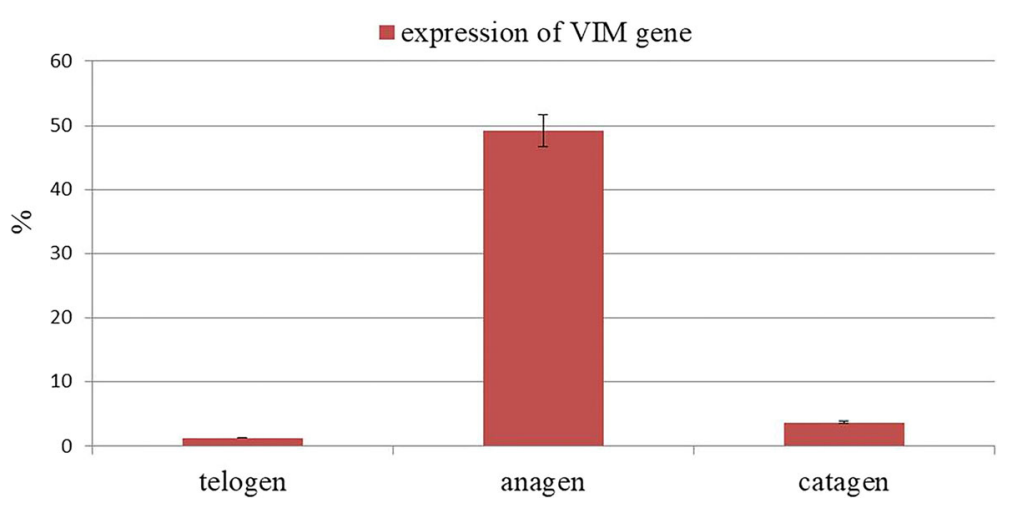

Fig. 3 Relative expression levels of the VIM gene in the stages of the skin follicle cycle. The RT-PCR was performed using the $2^{-\Delta \Delta C T}$ method. The values are shown as the mean \pm standard deviation. A significance level of 0.05 was used

into the dermis. After the predetermined depth is reached, the cells in the central cylinder reverse their growth direction and progress distally, forming the IRS and the hair shaft. Consecutively, the outer cells form the ORS and the dermal sheath. In catagen, the proliferation of the dermal papilla cells is decreased, the hair bulb atrophied, the hair papilla separated from other structures, and the hair follicle shortened. The telogen is not a vital process of cell proliferation, apoptosis, and differentiation. The paraffin section technique was used by $\mathrm{Li}$ et al. [28] to study the morphological changes of the Inner Mongolia Cashmere goat skin follicles. The study found that anagen occurred from April-November, catagen from December-January, and telogen from

\footnotetext{
Inner Yongolis casheere zost :

Capra hircus

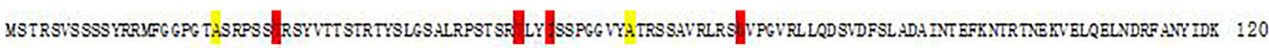

Capra hircus

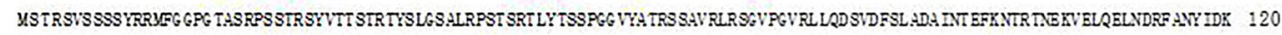

Bos Tourus

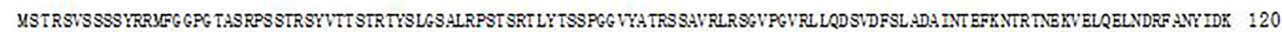

Hozo sapiens

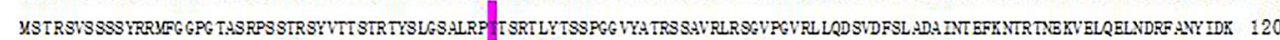

Yus ausculus

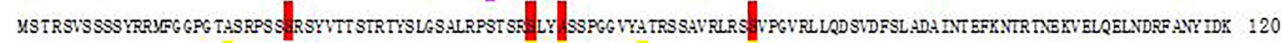

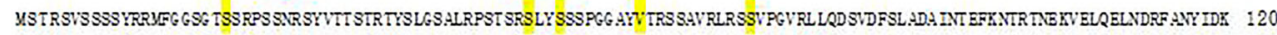

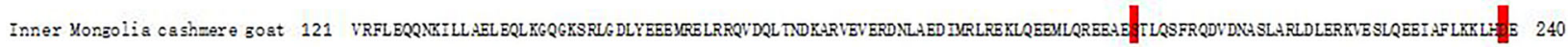

Capra hircus

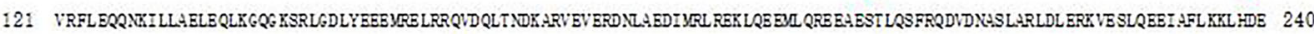

Fig. 4 Multiple sequence alignment of vimentin. The amino acid sequences of vimentin were analyzed by multiple sequence alignment. The blue mark represents the differentially expressed sequence of vimentin between Inner Mongolia Cashmere goat and Ovis aries. The red mark indicates the site with a difference from that of Homo sapiens, and the yellow mark represents the site with a difference in the sequence from that of Mus musculus 


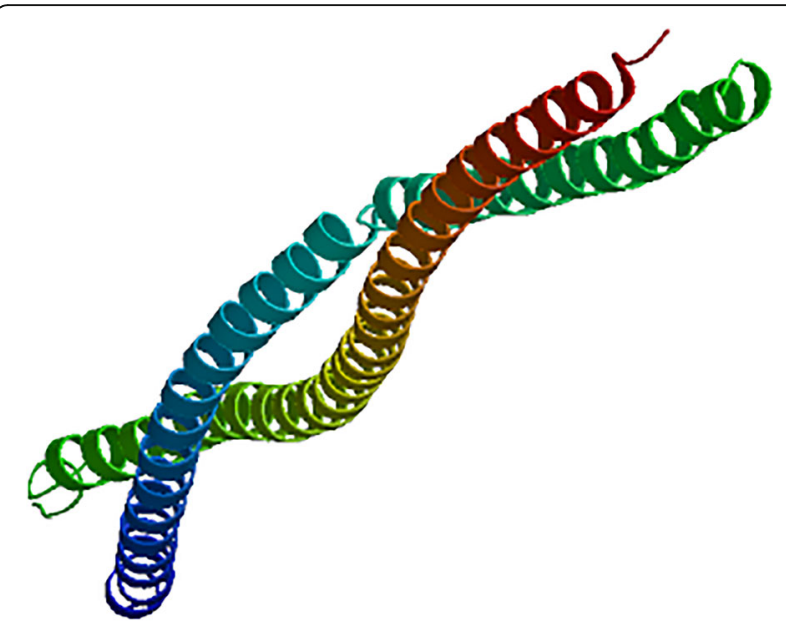

Fig. 5 Three-dimensional structure of vimentin. The three-dimensional structure of vimentin predicted based on the amino acid sequence

February-March, which indicates the duration of 8,2 , and 2 months, respectively. In this study, transcriptome sequencing and liquid chromatography-tandem mass spectrometry were used to obtain the nucleic acid and amino acid sequences of vimentin, and the comparison analysis used for the amino acid and nucleic acid sequences. RT-PCR, Western blot, and immunohistochemistry analyzed the expression level and expression sites of vimentin during the telogen, anagen, and catagen stages of the skin in Inner Mongolia Cashmere goat.

\section{Analysis of sequences and expression level of VIM gene and vimentin}

Vimentin is a type III IF protein, which might be secreted by invading macrophages [29] or derived from the adjacent dermal telocytes [30]. The primary function of vimentin was to maintain the cell morphology [6-8], transmit signals between cells [9-11], and regulate the cell differentiation and proliferation [12]. In addition, vimentin induces cell migration [31]. The cell culture experiments revealed that the presence of vimentin in fibroblasts was associated with $\alpha$-smooth muscle actin and myosin, and thus, the in vitro cultured fibroblasts also exhibit vimentin expression [32]. Notably, in the embryonic period, vimentin might participate in the adjustment of the arrangement of collagen fibers and inhibition of scar hyperplasia [33]. Furthermore, it is also involved in wound healing [34]. A previous study showed the highest expression of vimentin in the anagen of the growth cycle of the mouse skin follicle [21]. Another study revealed that vimentin was expressed at a maximum in the anagen of the Inner Mongolia Cashmere goat skin [22]. In the current study, the nucleic acid and amino acid sequences of vimentin in Inner Mongolian Cashmere goat skin sample were $100 \%$ identical to that of the Capra hircus. However, the rates of similarity to Ovis aries, Bos taurus, Homo sapiens, and Mus musculus were 99, 99, 98, and 97\%, respectively. These results did not show any difference among the varieties of vimentin and only a little difference was observed among the species. Thus, vimentin is highly conserved, as postulated by Ivaska et al. [5]. Furthermore, we investigated the expression of the VIM gene and vimentin protein by RT-PCR and Western blot, respectively. The trend of expression of VIM and vimentin was increased significantly in anagen, which was in agreement with previous studies $[21,22]$. A threedimensional analysis of the structure might provide an insight into the relationship between the structure and function of vimentin in future studies.

\section{Localization analysis of vimentin in the hair follicles}

Figure 4 demonstrated that vimentin was expressed only in the ORS of hair follicles. Therefore, the changes in the expression of vimentin and VIM genes in the skin samples should come from the ORS of the hair follicles. Thus, these results suggested that vimentin was involved in the regulation of the hair follicle growth cycle by affecting the ORS.

\section{Conclusions}

The sequence and comparative analysis of VIM gene and vimentin protein revealed that vimentin was highly

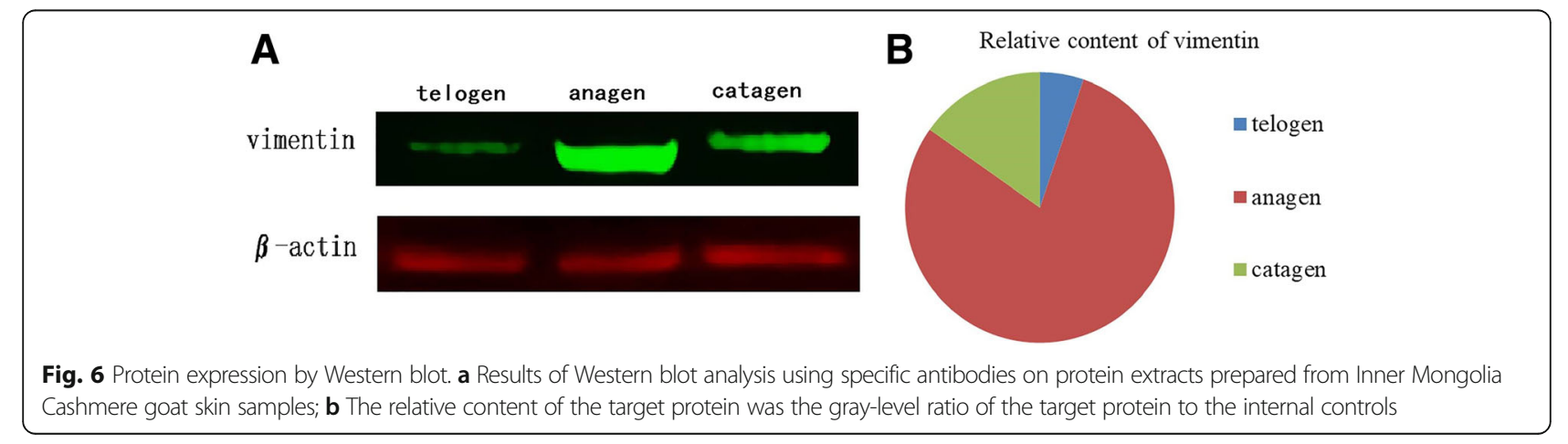



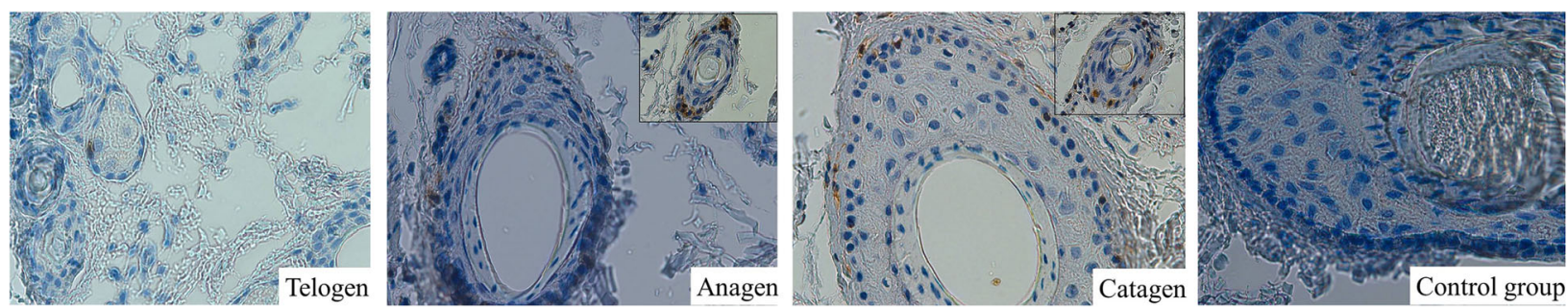

Fig. 7 Localization of vimentin in the hair follicle cycle by immunohistochemistry. Brown cells were considered to be positive. The big picture is the primary hair follicle. The small picture is the secondary hair follicle in the corresponding month

conserved across evolution. However, RT-PCR and Western blot demonstrated that the expression of VIM gene and vimentin increased with the growth of skin follicle and decreased with the decline in skin follicle growth. Immunohistochemistry showed that vimentin was expressed in the ORS of the hair follicle. Thus, we speculated that vimentin plays a role in the regulation of the hair follicle growth cycle by affecting the ORS.

\section{Abbreviations}

BSA: Albumin from bovine serum; H2O2: Hydrogen peroxide; IF: Intermediate filament; IRS: Inner root sheath; ORS: Outer root sheath; PBS: Phosphatebuffered saline; PBST: Phosphate-buffered saline with tween20; RT-PCR: Realtime quantitative PCR; SDS: Sodium dodecyl sulfate; SDS-PAGE: SDSpolyacrylamide gel electrophoresis

\section{Acknowledgements}

The authors are thankful for the samples provided by the Aerbasi White Cashmere Goat Breeding Farm. Jinquan Li provided the test platform, whereas Zhihong Liu helped in designing and conducting the experiments and the analysis, evaluation, and interpretation of the results.

\section{Funding}

This work has been funded under the National Natural Science Foundation of China (\#31360537, \#31660640), the National "863" Project (\#2013AA102506), the Natural Science Foundation of Inner Mongolia (\#2017MS0356), and the Major projects of Inner Mongolia Natural Science Foundation (\#2016ZD02). The Major projects of Inner Mongolia Natural Science Foundation (\#2016ZD02) and the Natural Science Foundation of Inner Mongolia (\#2017MS0356) provided support in the design of the study and collection. The National Natural Science Foundation of China (\#31360537, \#31660640) and the National "863" Project (\#2013AA102506) provided support in the analysis and interpretation of data and in writing the manuscript.

\section{Availability of data and materials}

The DNA sequence of Capra hircus VIM gene, obtained from NCBI/GENE (NCBI Reference Sequence is NC_030820.1) was compared against that of NCBI/ BLAST, and the RID was W5UR5P1114. The sequence of vimentin was obtained from Triple TOF 5600 (AB Sciex, USA) and Protein Pilot 4.0. The UniProt/Swiss-Prot database was used as the background, and the serial number was P48616. The comparative analysis of proteins was conducted in NCBI/BLAST, and the RID was YUG6J01Y014.

\section{Authors' contributions}

$N R$, ZL, LG, and JQ made substantial contributions to the conception and design of the experiments. Conceived and designed the experiments: $R S, Y Z$, RW, JL. Performed the experiments: NR, JQ, MZ, LG, YX. Analyzed the data: NR, $J \mathrm{~L}, \mathrm{ZL}$. Wrote the paper: NR, HX, ZL. Critically revised the manuscript: HX, ZL. All authors read and approved the final manuscript.

\section{Ethics approval}

All animal procedures were approved by the Inner Mongolia Agriculture University Animal Care Standard (GB 14925-2001). All experiments were performed in accordance with relevant guidelines and regulations. All efforts were made to minimize animal suffering.

\section{Consent for publication}

Not applicable.

\section{Competing interests}

The authors declare that they have no competing interests.

\section{Publisher's Note}

Springer Nature remains neutral with regard to jurisdictional claims in published maps and institutional affiliations.

\section{Author details}

${ }^{1}$ College of Animal Science, Inner Mongolia Agricultural University, Hohhot 010018, China. ${ }^{2}$ Key Laboratory of Animal Genetics, Breeding and Reproduction, Inner Mongolia Autonomous Region, Hohhot, China. ${ }^{3}$ Key Laboratory of Mutton Sheep Genetics and Breeding, Ministry of Agriculture, Hohhot, China. ${ }^{4}$ Engineering Research Center for Goat Genetics and Breeding, Inner Mongolia Autonomous Region, Hohhot, China. ${ }^{5}$ School of Life Science, Inner Mongolia University for The Nationalities, Tongliao 028000 China. ${ }^{6}$ Inner Mongolia Academy of Agriculture and Animal Husbandry Sciences, Hohhot 010018, China. ${ }^{7}$ Baotou Light Industry Vocational Technical College, Baotou 014000, China.

Received: 6 July 2017 Accepted: 22 December 2017

Published online: 10 January 2018

\section{References}

1. Zen Z. Study on the fur growth rules of inner Mongolian cashmere goats [D]: In Inner Mongolia. China: Inner Mongolia Agricultural University; 2011.

2. Coulombe PA, Wong P. Cytoplasmic intermediate filaments revealed as dynamic and multipurpose scaffolds.[J]. Nat Cell Biol. 2004;68:699-706.

3. Fuchs E, Weber K. Intermediate filaments: structure, dynamics, function, and disease.[J]. Annu Rev Biochem. 1994;63:345-82.

4. Steinert PM, Roop DR. Molecular and cellular biology of intermediate filaments[J]. Annu Rev Biochem. 1988:57:593-625.

5. Ivaska J, Pallari H-M, Nevo J, Eriksson JE. Novel functions of vimentin in cell adhesion, migration, and signaling[J]. Exp Cell Res. 2007:313(10):2050-62.

6. Yan-jun ZHANG, Jun YIN, Jin-quan LI, Chang-qing LI. Study on hair follicle structure and morphogenesis of the inner Mongolian Arbas cashmere goat [J]. Sci Agric Sin. 2007;05:1017-23.

7. Changqing LI, Jun YIN, Yanjun ZHANG, Zhicheng GUO, Wenguang ZHANG, Aiqin GAO, Yurong LI, Shuangying LAI, Jinquan LI. Comparative study on skin and hair follicles cycling between Inner Mongolia and Liaoning cashmere goats [J]. Chin J Anim Vet Sci. 2005;07:674-9.

8. Ansari-Renani HR, Ebadi Z, Moradi S, Baghershah HR, Ansari-Renani MY, Ameli SH. Determination of hair follicle characteristics, density and activity of Iranian cashmere goat breeds[J]. Small Rumin Res. 2010;952:128-32.

9. Li Q-F, Spinelli AM, Wang R, Anfinogenova Y, Singer HA, Tang DD. Critical role of vimentin phosphorylation at Ser-56 by p21-activated kinase in vimentin cytoskeleton signaling.[J]. J Biol Chem. 2006:281(45):34716-24.

10. Perlson E, Hanz S, Ben-Yaakov K, Segal-Ruder Y, Seger R, Fainzilber M. Vimentin-dependent spatial translocation of an activated MAP Kinase in injured nerve[J]. Neuron. 2005;45(5):715-26. 
11. Perlson E, Michaelevski I, Kowalsman N, Ben-Yaakov K, Shaked M, Seger R, Eisenstein M, Fainzilber M. Vimentin binding to Phosphorylated Erk Sterically hinders enzymatic Dephosphorylation of the Kinase[J]. J Mol Biol. 2006; 364(5):938-44.

12. Kero D, Kalibovic Govorko D, Vukojevic K, Cubela M, Soljic V, Saraga-Babic M. Expression of cytokeratin 8, vimentin, syndecan-1 and Ki-67 during human tooth development[]]. J Mol Histol. 2014;45(6):627-40.

13. Zhu QS, Rosenblatt $\mathrm{K}$, Huang $\mathrm{KL}$, et al. Vimentin is a novel AKT1 target mediating motility and invasion[J]. Oncogene. 2011;30(4):457-70.

14. Alexander DJ. Disease of poultry [M]: In lowa State. USA: lowa state University Press; 1997. p. 543

15. Sabbah M, Emami S, Redeuilh G, et al. Molecular signature and therapeutic perspective of the epithelial-to-mesenchymal transitions in epithelial cancers [J]. Drug Resist Updat. 2008;1 1(4-5):123-51.

16. Satelli A, Li S. Vimentin in cancer and its potential as a molecular target for cancer therapy [J]. Cell Mol Life Sci. 2011;68(18):3033-46.

17. Arri J, Goto H, Suenaga T, et al. Non-musclemyosin IIAIS a functional entry receptor for herpes simplex virus-1 [J]. Nature. 2010;467(7317):859-62.

18. Thomas EK, Connelly RJ, Pennathur S, et al. Antiidiotypic antibody to the V 3 domain of gp120 binds to vimentin: a possible role of intermediate filaments in the earlyesteps of HIV-1 infextion cycle []]. Viral Immunol. 1996;9(2):73-87.

19. Ivaska J, Pallari HM, Nevo $\mathrm{H}$, et al. Novel functions of vimentin in cell adhesion, migration, and signaling[J]. Exp Cell Res. 2007;313(10):2050-62.

20. Wang $\mathrm{Q}$, et al. The changing of type I, III collagen and vimentin expression in rat embryonic skin and its significance [J]. J Luzhou Med Coll. 2011;05:526-9.

21. Wang L, Xu W, Cao L, Tian T, Yang M, Li Z, Ping F, Fan W. Differential expression of proteins associated with the hair follicle cycle-proteomics and bioinformatics analyses [J]. PLoS One. 2016;11(1):e0146791.

22. Li-Xia GAO. Study on different proteomics of hair follicle cycle of Inner Mongolia Cashmere goat [D]: In Inner Mongolia. China: Inner Mongolia Agricultural University; 2014.

23. Qiao-Ling S. RAPD analysis of nucleus DNA in different-area main cashmere breeds (groups) in China [D]: In Inner Mongolia. China: Inner Mongolia Agricultural University; 2002.

24. Xin L. The research of human dermal papilla cells and outer root sheath cells interact with androgen [D]. Guangdong: Southern Medical University; 2006.

25. Inui S, Fukuzato Y, Nakajima T, Yoshikawa K, Itami S. Androgen-inducible TGF-b1 from balding dermal papilla cells inhibits epithelial cell growth: a clue to understand paradoxical effects of androgen on human hair growth. FASEB J. 2002;16:1967-9.

26. Stenn KS, Paus R. Controls of hair follicle cycling[J]. Physiol Rev. 2001; 81(1):449-94.

27. Paus R, Foitzik K, In search of the " hair cycle clock": a guid tour [J]. Differentiation. 2004;72(9-10):489-511.

28. Chang-ging LI. Hair follicles cycling of cashmere goats and $m$ RNA expression of KAP13-1 during Anagen [D]: In Inner Mongolia. China: Inner Mongolia Agricultural University; 2005

29. Mor-Vaknin N, Punturieri A, Sitwala K, Markovitz DM. Vimentin is secreted by activated macrophages. Nat Cell Biol. 2003;5(1):59-63.

30. Slominski A, Paus R, Plonka P, et al. Melanogenesis during the anagencatagen-telogen transformation of the murine hair cycle. J Invest Dermatol. 1994;102(6):862-9.

31. Ivaska J. Vimentin: central hub in EMT induction. Small GTPases. 2011;2(1): 51-3. PMID

32. Fuchs EC, Cleveland DW. A structural scaffolding of intermediate filaments in health and disease [J]. Science. 1998;279:514.

33. Zhen-xiang WANG, Jun WU, Shaoxuan YI, et al. Effect of vimentin expression on contraction of hypertrophic scar [J]. Chin J Med Aesthetics Cosmetology. 2003;9(5):293

34. Menko AS, Bleaken BM, Libowitz AA, Zhang L, Stepp MA, Walker JL. A central role for vimentin in regulating repair function during healing of the lens epithelium. Mol Biol Cell. 2014;25(6):776-90.

\section{Submit your next manuscript to BioMed Central and we will help you at every step:}

- We accept pre-submission inquiries

- Our selector tool helps you to find the most relevant journal

- We provide round the clock customer support

- Convenient online submission

- Thorough peer review

- Inclusion in PubMed and all major indexing services

- Maximum visibility for your research

Submit your manuscript at www.biomedcentral.com/submit
Biomed Central 